\title{
Diagnostic Test Validity of Oxacillin Screen Agar for the Detection of Methicillin Resistant Staphylococcus aureus
}

\author{
Hafiza Sultana ${ }^{*}$, Md. Abdullah Yusuf ${ }^{2}$, Jogendra Nath Sarker ${ }^{3}$, \\ Md. Tofael Hossain Bhuiyan" ${ }^{4}$, Shirin Tarafder ${ }^{5}$ \\ ${ }^{1}$ Department of Microbiology, Rangpur Medical College, Rangpur, Bangladesh \\ ${ }^{2}$ Department of Microbiology, National Institute of Neurosciences \& Hospital, Dhaka, Bangladesh \\ ${ }^{3}$ Department of Microbiology, Dinajpur Medical College, Dinajpur, Bangladesh \\ ${ }^{4}$ Department of Neurosurgery, Rangpur Medical College Hospital, Rangpur, Bangladesh \\ ${ }^{5}$ Department of Microbiology \& Immunology, Bangabandhu Sheikh Mujib Medical University, Dhaka, Bangladesh \\ Email: *dr.hafizasultana@yahoo.com, ayusuf75@yahoo.com, jogendrasarker@gmail.com, \\ drthbhuiyan@gmail.com, starafder2007@yahoo.com
}

\begin{abstract}
How to cite this paper: Sultana, H., Yusuf, Md.A., Sarker, J.N., Bhuiyan, Md.T.H. and Tarafder, S. (2018) Diagnostic Test Validity of Oxacillin Screen Agar for the Detection of Methicillin Resistant Staphylococcus aureus. Open Journal of Medical Microbiology, 8, 26-33.

https://doi.org/10.4236/ojmm.2018.82003
\end{abstract}

Received: February 7, 2018

Accepted: June 26, 2018

Published: June 29, 2018

Copyright $\odot 2018$ by authors and Scientific Research Publishing Inc. This work is licensed under the Creative Commons Attribution International License (CC BY 4.0).

http://creativecommons.org/licenses/by/4.0/

\section{(c) (i) Open Access}

\begin{abstract}
Background: Detection of Methicillin Resistant Staphylococcus aureus may vary in phenotypic and genotypic methods. Objective: The aim of this present study was to validate the oxacillin screen agar test for the detection of methicillin-resistant Staphylococcus aureus isolated from clinical specimens. Methodology: This cross-sectional study was planned to carry out in the Department of Microbiology and Immunology at Bangabandhu Sheikh Mujib Medical University, Dhaka from January, 2010 to December, 2010 for a period of one (01) year. Different clinical specimens were collected from the patients presented with infections at anybody sites. Isolation and identification of Staphylococcus aureus ( $S$. aureus) were performed by staining, culture and biochemical tests. Oxacillin screen agar test was performed to detect the methicillin-resistant Staphylococcus aureus. PCR was performed for detection of the $m e c A$ gene for MRSA. Result: Out of the 22 suspected MRSA isolates 19 were $m e c A$ positive by PCR. The sensitivity and specificity of oxacillin screen agar were $100.0 \%$ (95\% CI 82.3 to $100.0 \%$ ) and $33.3 \%$ (95\% CI 24.0 to $90.6 \%$ ) respectively. The positive predictive value and negative predictive value of oxacillin screen agar test were $90.5 \%$ (95\% CI 81.0 to $95.5 \%)$ and $100.0 \%$ (95\% CI 20.6 to $100.0 \%$ ) respectively. The accuracy of the oxacillin screen agar test was 90.9\% (95\% CI 71.7 to 96.3\%). Conclusion: In conclusion oxacillin screen agar test has high sensitivity with low specificity for the detection of MRSA.
\end{abstract}

\section{Keywords}

Validity Test, Cefoxitin Disc Diffusion Test, Methicillin-Resistant, 


\section{Introduction}

The prevalence of MRSA among clinical isolates varies from country to country and from institution to institution [1]. In Bangladesh, a multi-centre study [2] shows the rate of isolation of MRSA from hospital patients ranged between 32 to $63 \%$. Study by Zahan et al. [3] reported that, 15 (37.5\%) out of 40 isolated $S$. aureus strains were MRSA by oxacillin disc diffusion and agar dilution method but by PCR 10 (25\%) strains yielded mecA gene. These data show that screening of the suspected MRSA strain by oxacillin disc diffusion does not coincide with PCR closely which needs further study to find out other antibiotic or method which can be more reliable as a screening test.

The Clinical and Laboratory Standard Institute has recommended cefoxitin disc diffusion method for the detection of MRSA for primary screening [4]. Multiple reports on the use of cefoxitin as an accurate surrogate marker for detection of mecA-gene-mediated methicillin resistance have been published. Among them Anand et al. [5] reported a comparative result of $50 \mathrm{~S}$. aureus isolates using oxacillin screening agar and PCR which shows $100 \%$ agreement between PCR while $96.8 \%$ with oxacillin agar screen. Similarly, Mathew et al. [6] published their report on 610 strains of $S$. aureus. As seen here, out of 228 isolates of MRSA, mecA gene was positive in only 20 (3.2\%) cases which was confirmed by PCR. This discrepancy encourages a need for further study with oxacillin screen agar test to come to a conclusion. Batista et al. [7] reported another comparative study of 53 clinical isolates of $S$. aureus using VITEK-2 system, oxacillin screen agar test and PCR which shows oxacillin screen agar method fails to detect some MRSA population due to its heterogenous resistance. Therefore, this present study was undertaken to detect the MRSA by oxacillin screen agar method and to compare it with PCR for the detection of mecA gene for MRSA.

\section{Methodology}

This cross-sectional study was conducted in the Department of Microbiology and Immunology at Bangabandhu Sheikh Mujib Medical University, Dhaka from January 2010 to December 2010 for a period of twelve (12) months. Different clinical samples were collected from the patients at any age with both sexes which included wound swab, pus, blood, urine, tracheal aspirate, throat swab, sputum, aural swab, nasal swab, high vaginal swab, burn swab, drain fluid and fluid from pleural effusion. All specimens were collected aseptically from three hospitals, namely Bangabandhu Sheikh Mujib Medical University (BSMMU), Dhaka Medical College Hospital (DMCH), Dhaka and a private diagnostic center in Dhaka. The samples were inoculated into appropriate media and were incubated aerobically at $37^{\circ} \mathrm{C}$ for 24 hours. Then colonies were identified for Sta- 
phylococcus species and were confirmed by Gram staining, colony morphology, haemolytic status, pigment production, mannitol fermentation test, motility test (MIU) and other relevant biochemical tests like catalase test, coagulase test both slide and tube test as per standard procedure [8]. In this study, screening for MRSA was done by oxacillin and oxacillin screening agar [6]. In case of oxacillin the diameter of zone of inhibition $\leq 10 \mathrm{~mm}$ was taken as resistant [9]. Conventional PCR was performed to detect mecA gene of 22 suspected $S$. aureus strains resistant to oxacillin by disc diffusion method at the Molecular Laboratory in the Department of Microbiology and National Forensic DNA Profiling Laboratory of Dhaka Medical College, Dhaka. Methicillin-resistant $S$. aureus (MRSA) strain [ATCC 43300] were used as positive control. PCR for mecA gene detection were performed by formation of bacterial pellet, DNA extraction, preparation of reaction mixture $(25 \mu \mathrm{l})$ and running in thermo cycler. Primers used for detection of the $m e c A$ gene producing a 309-bp amplicon were as follows:

mecA1-F-5 'TGGCTATCGTGTCACAATCG 3' (positions 885 to 905) and

mecA2-R-5'CTGGAACTTGTTGAGCAGAG 3'(positions 1174 to 1194)

PCR reactions were performed in a thermocycler under the following conditions: initial denaturation for 10 minutes at $94^{\circ} \mathrm{C}$ followed by 30 cycles at $94^{\circ} \mathrm{C}$ for 1 minute, at $54^{\circ} \mathrm{C}$ for 1 minute, then at $72^{\circ} \mathrm{C}$ for 1 minute. Final extension was for 7 minutes at $72^{\circ} \mathrm{C}$. Mixed the amplicon and ladder with dye (4-5:1 ratio). Then pipetting and dispensing were done onto the wells on gel made by comb. Start the gel electrophoresis at 100 volt for 60 minutes until the end of the reaction indicated by orange color advancement was over. Ethidium bromide $(7.5 \mu \mathrm{l})$ mixed with distilled water $(100 \mathrm{ml})$. Gel was placed in this mixture for $30 \mathrm{mi}-$ nutes staining. Again destaining done in pure water for 20 minutes. The destained gel was placed on UV transilluminator and observed for the presence of DNA bands. Gels were visualized and photographed under ultraviolet illumination. Precautions were taken to prevent the samples from being contaminated by each other or by the skin of laboratory personnel.

\section{Results}

A total of 120 staphylococcus aureus (S. aureus) isolates were collected from 266 specimens from three different hospitals over a period of January, 2010 to December, 2010. Of which 80 (66.7\%) S. aureus were from Bangabandhu Sheikh Mujib Medical University (BSMMU), 10 (8.3\%) from Dhaka Medical College Hospital (DMCH) and rest 30 (25.0\%) were collected from Popular Diagnostic Center (PDC), Dhaka. These isolates were subjected to antimicrobial susceptibility testing by oxacillin screen agar test for detection of phenotypical detection of MRSA and PCR for detection of the mecA gene. Among the 22 Staphylococcus aureus isolates 21 (95.5\%) isolates were oxacillin screen agar resistant indicating that these are MRSA and the rest 1 (4.5\%) isolate was methicillin sensitive Staphylococcus aureus (MSSA). However, mecA gene was positive by PCR in 19 (100.0\%) Staphylococcus aureus isolates out of 21 isolates $(\mathrm{p}=0.01)($ Table 1$)$. 
Table 1. Comparison of oxacillin screen agar with PCR results on suspected MRSA isolates $(n=22)$.

\begin{tabular}{ccccc}
\hline \multirow{2}{*}{ Oxacillin screen agar } & \multicolumn{2}{c}{ PCR Results } & \multirow{2}{*}{ Total } & P value \\
\cline { 2 - 3 } & Positive & Negative & & \\
\hline Resistant & $19(100.0 \%)$ & $2(66.7 \%)$ & $21(95.5 \%)$ & \\
Sensitive & $0(0.0 \%)$ & $1(33.3 \%)$ & $1(4.5 \%)$ & 0.01 \\
Total & $19(100.0 \%)$ & $3(100.0 \%)$ & $22(100.0 \%)$ & \\
& & &
\end{tabular}

The sensitivity and specificity of oxacillin screen agar were $100.0 \%$ (95\% CI $82.3 \%$ to $100.0 \%$ ) and $33.3 \%$ (95\% CI $24.0 \%$ to $90.6 \%$ ) respectively. The positive predictive value and negative predictive value of oxacillin screen agar test were 90.5\% (95\% CI $81.0 \%$ to $95.5 \%$ ) and $100.0 \%$ (95\% CI $20.6 \%$ to $100.0 \%$ ) respectively. The accuracy of the oxacillin screen agar test was $90.9 \%$ (95\% CI $71.7 \%$ to 96.3\%) (Table 2).

The receiver operative characteristics (ROC) curve was calculated and had found that the value of area under the curve (AUC) was low which was 0.33 with a $95 \%$ CI 0.0001 to $0.727(\mathrm{p}=0.363$ ) (Figure 1).

\section{Discussion}

The methods that stand out among the most utilized or best capable in the identification of methicillin-resistant Staphylococcus aureus (MRSA) are agar dilution method, disk diffusion, screening on agar with methicillin, automated methods (Microscan, Vitek), latex agglutination and the molecular method using PCR for the detection of the gene mecA [8] The polymerase chain reaction in real time (RT-PCR) was described as a useful PCR-based diagnostic tool [9] [10].

Approximately $20.0 \%$ of healthy persons are persistent carriers of $S$. aureus and 60 percent are intermittent carriers. Colonization rates are increased in haemodialysis patients, illicit injecting drug users, surgical patients and patients with insulin dependence or poorly controlled diabetes [11]. Healthcare associated infection are leading causes of morbidity \& mortality [12] and the management of all these condition has been critically compromised by the appearance \& rapid spread of antimicrobial resistance among the organisms floating in the hospital. Therefore, the importance of MRSA detection especially for

Table 2. Diagnostic test validity of oxacillin screen agar in diagnosis of MRSA.

\begin{tabular}{ccc}
\hline Variables & Values & $95 \%$ CI \\
\hline Sensitivity & $100.0 \%$ & $82.3 \%$ to $100.0 \%$ \\
Specificity & $33.3 \%$ & $24.0 \%$ to $90.6 \%$ \\
PPV & $90.5 \%$ & $81.0 \%$ to $95.5 \%$ \\
NPV & $100.0 \%$ & $20.6 \%$ to $100.0 \%$ \\
Accuracy & $90.9 \%$ & $71.7 \%$ to $96.3 \%$
\end{tabular}

PPV—-positive predictive values; NPV—negative predictive values. 


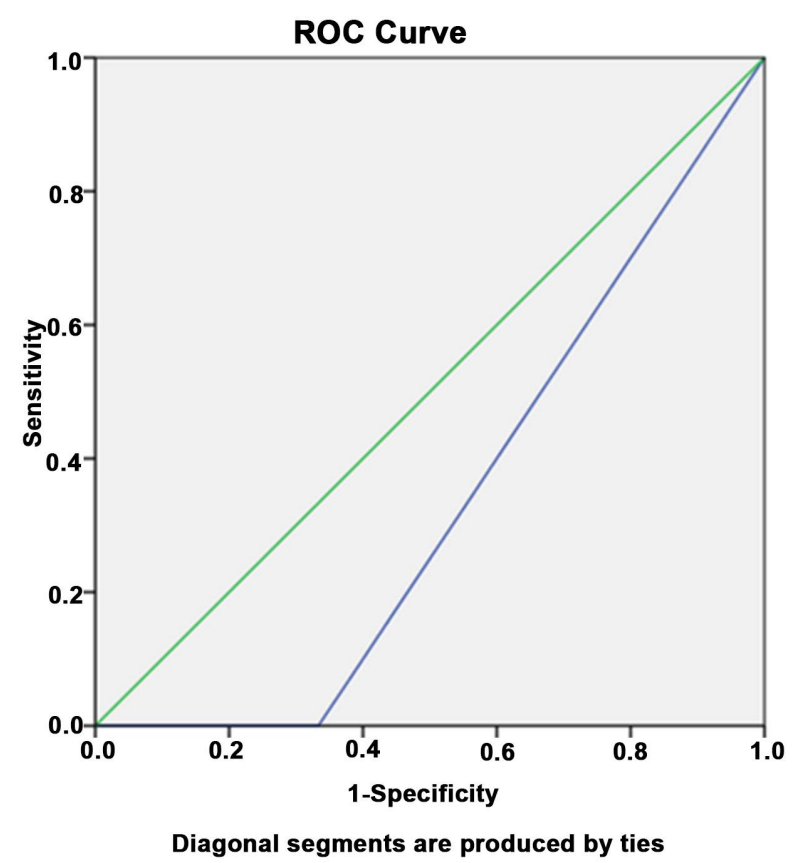

Figure 1. ROC curve with the value of area under the curve (value $=0.333,95 \%$ CI 0.0001 to 0.727 ; value $=0.363$ ) .

therapeutic \& epidemiological purpose is an acute need. Hence, the methods used to detect MRSA in clinical samples should have high sensitivity and specificity and most importantly the result should be available within a short time [13].

In this study, the number of suspected MRSA isolates by phenotypic methods was 22. Of these suspected cases 21 (95.5\%) were detected MRSA by oxacillin screen agar while it was $19(86.4 \%)$ by PCR for mecA gene. Identification of the mecA gene is the most reliable method of detecting MRSA isolates, however not all laboratories can include molecular biology techniques in their routine clinical practice. For this reason, it is essential that phenotypic techniques able to detect MRSA isolates in a rapid and accurate manner are made available, in order to ensure correct antibiotic treatment and to avoid the spread of MRSA isolates in the hospital environment.

In the present study comparison of oxacillin screen agar were evaluated for screening of MRSA. Of total 22 suspected isolates 19 were mecA positive by PCR which is "gold standard". Out of these 19 , all were also resistant on oxacillin screen agar. The 3 strains of $S$. aureus negative by PCR for mecA gene, of which $2(66.7 \%)$ were resistant on oxacillin screen agar. In the present study the sensitivity and specificity of oxacillin screen agar $100.0 \%$ and $33.3 \%$ respectively. Sensitivity and specificity of oxacillin screen agar which were reported by Mathew et al [6] were $97.5 \%$ and $75 \%$ respectively and by Anand et al. [5] were $96.8 \%$ and $100 \%$ respectively in their study in which sensitivity was almost similar to the present study. Baddour et al. [11] described several conventional methods to detect MRSA and were compared with polymerase chain reaction (PCR) for de- 
tection of $\operatorname{mec} A$ gene-positive isolates. Oxacillin agar screen methods were found to be more sensitive than oxacillin disc-diffusion methods which were not with the similar statement with our study.

Various other studies conducted in Bangladesh indicate an increasing trend of MRSA all over the country. A multi-center study involving four divisional hospitals reported a MRSA rate range between $32 \%$ to $63.0 \%$ [2]. Study on patients of DMCH by Afroz [9], from NITOR (National institute of Traumatology and orthopedics Rehabilitation) and by Jahan et al. [4] reported a very high prevalence of MRSA which were $70.2 \%$ and $83.3 \%$ respectively. Such a high rate may be due to the specimen from post-operative wounds where the nosocomial infection is common. Other reasons may be small size of sample, severity of illness of the cases and the most important being that the above studies were based on susceptibility testing not confirmed by PCR. It seems that susceptibility testing for MRSA by oxacillin should only be treated as a screening test as Zahan et al. [3] reported $25 \%$ MRSA by PCR while it was $37.5 \%$ by oxacillin susceptibility testing from Mymensingh Medical College. Therefore, it is the time to take necessary measures to prevent the spread of MRSA by proper antibiotic policy, preventing nosocomial infection and proper detection and treatment of MRSA cases.

The present study indicates that the rate of MRSA among patients is moderate $(15.8 \%)$ in nature. The laboratory technique that is evaluated in this study, the sensitivity and specificity of oxacillin screen agar varies from PCR which means it is highly sensitive but low specificity and may be helpful tool in the diagnosis of MRSA infection. In addition to that as MRSA were highly resistant to common antimicrobial agents accurate detection is very important [13]. Therefore, it is necessary that antimicrobial susceptibility test on all MRSA isolates should be done to promote the rationale use of drugs and combat spread of MRSA.

AUC is an effective way to summarize the overall diagnostic accuracy of the test. It takes values from 0 to 1 , where a value of 0 indicates a perfectly inaccurate test and a value of 1 reflects a perfectly accurate test. AUC can be computed using the trapezoidal rule [12]. In general, an AUC of 0.5 suggests no discrimination which indicates that the ability to diagnose patients with and without the disease or condition based on the test; 0.7 to 0.8 is considered acceptable, 0.8 to 0.9 is considered excellent, and more than 0.9 is considered outstanding. A value of 0.5 for AUC indicates that the ROC curve will fall on the diagonal that is 45-degree line and hence suggests that the diagnostic test has no discriminatory ability [13]. ROC curves above this diagonal line are considered to have reasonable discriminating ability to diagnose patients with and without the disease/condition. It is therefore natural to do a hypothesis test to evaluate whether the AUC differs significantly from 0.5.

\section{Conclusion}

In conclusion oxacillin screen agar test is highly sensitive for the detection of MRSA; however this test has a very low specificity in comparison to $\operatorname{mec} A$ gene 
detection by PCR. Furthermore the area under the curve is low which indicates that the accuracy of oxacillin screen agar test is not a suitable test for the detection of MRSA from the clinical specimens.

\section{Contribution to Author}

Hafiza Sultana, Jogendra Nath Sarker and Shirin Tarafder were involved from protocol preparation, data collection, analysis and reort writing. Md. Abdullah Yusuf and Md. Tofael Hossain Bhuiyan were contributed in the manuscript preparation and revision.

\section{Conflict of Interest}

There was no conflict of interest to any of the authors related to financial matter.

\section{References}

[1] Anwar, M.S., Jaffery, G., Bhatti, K.U., Tayyab, M. and Bokhari, S.R. (2004) Staphylococcus aureus and Nasal Carriage in General Population. Journal of the College of Physicians and Surgeons Pakistan, 14, 661-664.

[2] Haq, J.A. (2009) Emerging Bacterial Resistance to Antibiotics-Fighting a Losing Battle! Ibrahim Medical College Journal, 3, i-ii. https://doi.org/10.3329/imcj.v3i1.2908

[3] Zahan, N.A., Hossain, M.A., Musa, A.K., Shamsuzzaman, A.K., Mahamud, M.C., Mamun, A.A., et al. (2009) PCR for mecA Gene of Methicillin-Resistant Staphylococcus aureus. Mymensingh Medical Journal, 18, 21-26.

[4] Jahan, Y., Jahan, F., Mamun, K.Z., Hossain, M.A., Shirin, T., Sahman, S., et al. (2004) Emergence of Methicillin-Resistant Staphylococcus aureus (MRSA) Associated with Wound Infections. Mymensingh Medical Journal, 13, 76-81.

[5] Anand, K.B., Agrawal, P., Kumar, S. and Kapila, K. (2009) Comparison of Cefoxitin Disc Diffusion Test, Oxacillin Screen Agar and PCR for mecA Gege for Detection of MRSA. Indian Journal of Medical Microbiology, 27, 27-29.

[6] Mathew, A.A., Thomas, M., Appalaraju, B. and Jayalaxmi, J. (2010) Evaluation and Comparison of Tests to Detect Methicillin-Resistant Staphylococcus aureus. Indian Journal of Pathology \& Microbiology, 53, 79-82. https://doi.org/10.4103/0377-4929.59189

[7] Batista, N.D., González, G., Pérez, M.L., Laich, F. and Álvarez, S.M. (2008) Evaluation of the Cefoxitin $30 \mu \mathrm{g}$ Disc Diffusion Method for Detection of Methicillin-Resistance in Selected Staphylococcus aureus Isolates. Revista Espanola De Quimioterapia, 21, 213-216.

[8] Velasco, D., del MarTomas, M., Cartelle, M., Beceiro, A., Perez, A., Molina, F., et al. (2005) Evaluation of Different Methods for Detecting Methicillin (Oxacillin) Resistance in Staphylococcus aureus. Journal of Antimicrobial Chemotherapy, 55 379-382. https://doi.org/10.1093/jac/dki017

[9] Afroz, S. (2005) Detection of MRSA in Patients and Carriers by Evaluating Different Methods of Identification, Its Typing and Susceptibility to Vancomycin. M.Phil. Thesis, Department of Microbiology, Dhaka Medical College, Dhaka.

[10] Moreillon, P., Que, Y.A. and Glauser, M.P. (2005) Staphylococcus aureus (Including Staphylococcal Toxic Shock). In: Mandell, G.L., Bennett, J.E. and Dolin, R., Eds. Mandell, Douglas and Bennett's Principles and Practice of Infectious Disease, 6th 
Edition, Elsevier Livingstone, Philadelphia, 2321-2351.

[11] Baddour, M.M., AbuEIKheir, M.M. and Fatani, A.J. (2007) Comparison of Polymerase Chain Reaction with Phenotypic Methods for the Detection of Methicillin-Resistant Staphylococcus aureus. Current Microbiology, 55, 473-479. https://doi.org/10.1007/s00284-007-9015-6

[12] Rosner. B. (2005) Fundamentals of Biostatistics. 6th Edition, Duxbury, Belmont, 64-66, Chapter 3.

[13] Hosmer. D.W. and Lemeshow, S. (2000) Applied Logistic Regression. 2nd Edition, John Wiley and Sons, New York, Chapter 5, 160-164.

https://doi.org/10.1002/0471722146 